\title{
Atividade Física e Saúde nos Programas de Pós-Graduação no Brasil: breve análise a partir de Tomas Kuhn
}

\author{
Physical Activity and Health in Post-graduate courses: Analysis Supported \\ in the Thomas Kuhn`s ideas
}

\author{
Mathias Roberto Loch ${ }^{1,2}$ \\ 1. Departamento de Educação Física, \\ Universidade Estadual de Londrina, \\ Paraná, Brasil. \\ 2. Doutorando em Saúde Coletiva. \\ Universidade Estadual de Londrina, \\ Paraná, Brasil.
}

\begin{abstract}
Resumo
O objetivo deste ponto de vista foi refletir sobre a "Atividade Física e Saúde" no Brasil, especialmente nos programas de Pós Graduação Stricto Sensu, apoiado nas idéias de Tomas Kuhn. Buscando elementos empíricos para dar suporte ao ponto de vista, investigou-se a descrição das áreas de concentração e linhas de pesquisa em "Atividade Física e Saúde" (ou denominações semelhantes) dos Programas de mestrado e doutorado em Educação Física, Ciências da Atividade Física, Ciências da Motricidade e Ciências do Movimento Humano. O texto foi dividido em três partes: na primeira fez-se uma breve introdução à obra de Kuhn, especificamente sobre as observações do autor de como se dá o processo de desenvolvimento científico. Na segunda são apresentadas algumas reflexões sobre a situação da "Atividade Física e Saúde" no Brasil, principalmente a partir da constatação da heterogeneidade da descrição das áreas de concentração ou linhas de pesquisa em "Atividade Física e Saúde" dos Programas de Pós-Graduação investigados. Enquanto existem programas mais identificados com as ciências biológicas, outros parecem fundamentalmente buscar o entendimento das questões comportamentais e sociais ligadas à atividade física. Por fim, busca-se a síntese de algumas questões discutidas ao longo do trabalho. Espera-se que este texto demonstre que existe na "Atividade Física e Saúde" uma interessante possibilidade de convivência entre diferentes olhares, que pode inclusive fazer com que a área cresça de maneira mais sólida. Por outro lado, reconhecer e lidar com estas diferenças irá exigir maturidade e representa ainda um importante desafio.
\end{abstract}

Palavras- chave: Epistemologia; Atividade Física; Pós-Graduação; Paradigma; Educação Física.

\section{ENDEREÇOPARA CORRESPONDÊNCIA}

Mathias Roberto Loch Departamento de Educação Física Centro de Educação Física e Esporte Universidade Estadual de Londrina Rod. Celso Garcia Cid PR 445 Campus Universitário Londrina-PR 86051-990 - Caixa Postal 6001 mathias@uel.br

$\begin{array}{ll}\text { • Recebido: } & 06 / 10 / 2011 \\ \text { • Re-submissão: } & 16 / 11 / 2011 \\ & 25 / 11 / 2011 \\ \text { - Aceito: } & 27 / 11 / 2011\end{array}$




\section{INTRODUÇÃO}

Este texto é fundamentalmente um ponto de vista, baseado em reflexões e inquietações, e busca apoiar-se em alguns pontos da obra de Tomas Kuhn. De modo a fornecer elementos empíricos que dessem suporte ao ponto de vista, buscou-se investigar a descrição das áreas de concentração e linhas de pesquisa em "Atividade Física e Saúde" (ou denominações semelhantes) dos Programas de Mestrado e Doutorado em Educação Física reconhecidos pela Coordenação de Aperfeiçoamento de Pessoal de Nível Superior (CAPES). Assim, o objetivo foi refletir sobre a área "Atividade Física e Saúde" no Brasil, especialmente nos programas de Pós-Graduação Stricto Sensu, buscando levantar questões pertinentes ao momento atual da área, apoiado nas idéias de Kuhn. Para melhor organização do texto, optou-se pela divisão em três partes. A primeira introduz brevemente alguns pontos importantes na obra de Kuhn. A segunda apresenta a descrição das áreas/linhas de pesquisa em "Atividade Física e Saúde" nos Programas de Pós Graduação Stricto Sensu, e aborda o que denomino de paradigma centrado na aptidão física/exercício físico e o paradigma centrado na atividade física, além de realizar alguns comentários sobre a produção em "Atividade Física e Saúde" no contexto brasileiro. Por fim, busca-se a síntese de elementos discutidos ao longo do texto, bem como são realizadas algumas considerações sobre o futuro desta área no Brasil.

\section{Sobre a obra de Tomas Kuh: uma breve introdução}

Com formação inicial em física, Tomas Kuhn (1922-1996) é considerado um dos mais influentes pensadores da história e filosofia da ciência. Suas observações contribuíram no entendimento do processo que leva ao desenvolvimento científico. Para Kuhn, as características sociológicas das comunidades científicas são determinantes importantes do progresso científico, principalmente no que se refere ao abandono de uma estrutura teórica e sua substituição por outra.

Entre os vários conceitos explorados por Kuhn, possivelmente o mais importante seja o de paradigma. Sobre este conceito o autor menciona (2000, p. 219) que "é aquilo que os membros de uma comunidade científica partilham e, inversamente, uma comunidade científica consiste em homens que partilham um paradigma"1

Pode-se resumir a maneira como progride a ciência, para Kuhn, no seguinte esquema aberto (Figura 1). Esta sequência lógica foi construída por Kuhn a partir da observação do impacto das obras de, por exemplo, Newton, Darwin e Einstein, em suas respectivas áreas de conhecimento.

O período de "pré-ciência" é marcado pela: 1 - existência de muitas escolas; 2 - alguma realização científica notável; 3 - a redução do número de escolas (em geral para uma única); 4 - o início de uma prática científica mais eficiente.

Após este $4^{\circ}$ período, passa a ser hegemônica o que o autor denomina de ciência normal, que é eficiente para resolver problemas definidos dentro do seu paradigma, mas não é adequada para solucionar problemas que não se "encaixem" neste. Para Kuhn o cientista normal não consegue ser crítico do paradigma em que trabalha, pois se limita a concentrar esforços na articulação detalhada do paradigma no qual faz parte, e por vezes perde inclusive o senso crítico sobre as limitações de suas produções.

Outro conceito importante é o de crise, que é resolvida quando surge um paradigma novo que atrai a adesão de um número crescente de cientistas, até que eventualmente o paradigma original é abandonado. Para Kuhn, normalmente as revoluções são induzidas pelas crises, que em geral são uma consciência comum de que "algo saiu errado".

Com estas observações, Kuhn questiona a concepção que o progresso científico acontece pelo simples acúmulo de conhecimento e defende que a evolução se dá pelo que chama de processo de revolução científica. Assim, o progresso através de revoluções é a alternativa kuhniana para o progresso cumulativo característico dos relatos indutivistas da ciência, para o qual o conhecimento científico cresce continuamente à medida que observações mais numerosas e variadas são realizadas ${ }^{2}$. Popper ${ }^{3}$, outro importante filósofo da ciência, já havia avançado nesta observação, ao apontar que a evolução do conhecimento científico se dá à medida que conhecimentos anteriores são refutados, ao invés de acontecer pelo mero acúmulo.

Em relação à adesão dos cientistas à algum paradigma, para Kuhn, esta não acontece exclusivamente pelo convencimento (o cientista " $X$ " está convencido de que a teoria A é melhor do que B), mas muitas vezes se dá por questões principalmente pragmáticas (por exemplo, a possibilidade de um maior número de publicações, e uma consequente maior pontuação nos currículos). A "migração" de paradigma acontece de maneira similar. Nas palavras do próprio Kuhn (2000, p. 249): "se o novo ponto de vista perdura por algum tempo e continua a dar frutos, os resultados das pesquisas que podem ser verbalizados dessa forma crescem provavelmente em número. Eles (os cientistas normais) poderão dizer: não sei como os adeptos do novo ponto de vista tiveram êxito, mas preciso aprender; o que quer que estejam fazendo, é evidentemente correto. Essa reação ocorre mais facilmente entre os que acabam de ingressar na profissão, porque ainda não adquiriram o vocabulário e os compromissos especiais de qualquer um dos grupos"1.

Feita esta breve introdução à obra de Kuhn, e considerando que este pode fornecer elementos importantes para a reflexão sobre a área da "Atividade Física e Saúde" no Brasil, a seguir serão discutidos pontos considerados pertinentes, e buscar-se-á elementos em Kuhn para uma interpretação de alguns aspectos dessa área.

Reflexões sobre a "Atividade Física e Saúde" no Brasil

a) O paradigma centrado na Aptidão Física e no Exercício Físico e o paradigma centrado na Atividade Física

Considerando que a Educação Física, enquanto área/ campo acadêmico não possui unidade epistemológica ${ }^{4-8}$, não é de se estranhar que as suas sub-áreas (como a "Atividade Física e Saúde") apresentem também uma grande heterogeneidade.

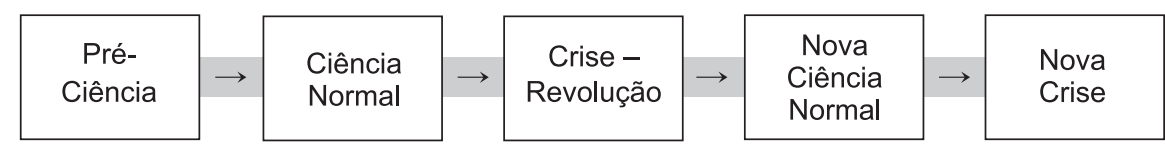


Há de se destacar nos últimos anos a evolução da pesquisa em "Atividade física e Saúde" no Brasil, especialmente na área da epidemiologia da Atividade Física9, 10, que não é apenas numérica, mas também importante qualitativamente.

A "Atividade Física e Saúde", enquanto uma área/sub-área/linha de pesquisa, parece assumir um significado bastante genérico, sendo praticamente um conceito "guarda chuva", onde quase tudo cabe, desde estudos que investigam efeito de programas formais e bem controlados de exercícios físicos sobre desfechos biológicos (com humanos e com animais), até estudos sobre a influência de determinantes sociais, culturais e ambientais sobre a prática ou não de atividades físicas. Ou seja, se por um lado, existem estudiosos da "Atividade Física e Saúde" preocupados essencialmente com aspectos biológicos, outros irão fundamentalmente buscar o entendimento de questões mais amplas ligadas à pratica de atividade física. Muitas vezes esta diferenciação passa despercebida pelos críticos, que na maioria das vezes rapidamente rotulam todas as produções dessa área como sendo oriundas das ciências biológicas.

Para exemplificar esta falta de homogeneidade na "Atividade Física e Saúde", foi realizada uma busca nos programas de Pós-Graduação Strito Sensu em Educação Física, Ciências da Atividade Física, Ciências da Motricidade e Ciências do Movimento Humano, reconhecidos pela CAPES ${ }^{11}$. Uma vez identificados os programas, visitou-se o sítio eletrônico de todos, e constatou-se que 17 (de 23) programas apresentam "Atividade Física e Saúde" (ou alguma denominação semelhante) como área de concentração ou linha de pesquisa. O quadro 1 apresenta maiores detalhes sobre as características e descrições destas áreas/linhas.

As informações descritas no Quadro 1 permitem constatar a grande diversidade nas abordagens relacionadas à "Atividade Física e Saúde". Em três programas (UNIVERSO, UCB e USJT) aparentemente há uma clara divisão entre a lógica centrada nas ciências biológicas e a centrada nas ciências sociais e/ou comportamentais. Seis programas (UNESP, UNICSUL, UNB, UFRN, UNIMEP E UEL/UEM) parecem apresentar ênfase no aspecto biológico. Outras seis instituições (UFES, UFMT, UFPR, UFSC, UFPEL e UPE/UFPB) apresentam descrições da área ou linha "Atividade Física e Saúde" que abrangem predominantemente os aspectos sociais e/ou comportamentais. Em duas instituições (UFRGS e UDESC), dada a descrição genérica das linhas, não foi possível caracterizar a predominância do foco dos respectivos programas.

É claro que a mera descrição das áreas e linhas não é suficiente para determinar com precisão a característica de cada um dos Programas investigados. Nem é este o objetivo do estudo. Para tal, uma análise mais detalhada, que incluísse, por exemplo, as dissertações e teses defendidas seria necessária. Para este texto, a apresentação da descrição das áreas e linhas objetivou mostrar a diversidade existente entre os programas. Neste sentido, confirmada esta diversidade, considero pertinente a divisão didática em dois paradigmas (mesmo reconhecendo a inter-relação entre ambos):

- Paradigma centrado na aptidão física e no exercício físico - Neste caso, os pesquisadores estão mais acostumados à lidar com situações clínicas. A preocupação com a validade interna dos estudos é preponderante. A exposição (ou variável independente) normalmente é a prática de exercícios físicos e os desfechos (variável dependente) normalmente estão relacionados à aptidão física ou à algum outro aspecto biológico. A pergunta central a ser respondida normalmente é: qual o efeito do exercício físico em diferentes indicadores de saúde?
Neste caso, normalmente os conhecimentos das ciências biológicas são os mais importantes.

- Paradigma centrado na atividade física - Neste caso, os pesquisadores apresentam uma maior preocupação com o mundo real (e não com situações de laboratório), sendo a preocupação com a validade externa destacada. A atividade física é entendida na maioria das vezes como desfecho (variável dependente) e tem como questão central o entendimento do comportamento humano. Neste caso as ciências humanas e comportamentais deveriam ser as mais relevantes.

Também na epidemiologia, Carvalho (2006) ${ }^{12}$ destaca a existência de duas "lógicas" (que também apresentam inter-relações importantes): uma hegemonicamente clínica, que está prioritariamente interessada em explicar por que e como um determinado sujeito adquiriu certa doença/agravo, e outra epidemiológica, que não é construída para falar da especificidade do caso individual, mas busca compreender as doenças/agravos de saúde como integrantes de uma determinada estrutura social.

Parece existir uma maior aproximação do paradigma da aptidão física/exercício físico com a denominada lógica clínica, enquanto que o paradigma da atividade física guarda uma maior relação com a lógica epidemiológica. Do mesmo modo, os Programas que apresentaram ênfase no aspecto biológico parecem estar mais próximos do paradigma da aptidão física e do exercício físico, e os com maior relação com os aspectos culturais, sociais e/ou comportamentais com o paradigma da atividade física.

Em muitas situações os paradigmas da atividade física e da aptidão física/exercício físico podem ter uma forte inter-relação. Por exemplo: um estudo de coorte que busca verificar a relação entre a prática de exercícios físicos ao longo da vida e a incidência de hipertensão. Neste caso, possivelmente existam elementos do paradigma da aptidão física e do exercício físico (pode se querer responder questões ligadas ao volume, intensidade, tipo e frequência dos exercícios realizados para se identificar os pontos que diminuem a incidência da hipertensão) e do paradigma da atividade física (por exemplo, se investigando os fatores associados à prática da atividade física ao longo da vida).

Saliento que não se faz aqui juízo de valor sobre a menor ou maior importância de um ou outro paradigma. Apenas reforço que existem importantes diferenças, inclusive no que se refere à fundamentação que deve ser utilizada em cada caso.

Além do mais, o problema a priori não está na heterogeneidade da "Atividade Física e Saúde". Na verdade o entendimento de objetos complexos (como é o caso) pode ser enriquecido com esta "diversidade de olhares". Por outro lado, o referencial conceitual/teórico utilizado precisa ser coerente com o objeto estudado. Por exemplo: alguns pesquisadores com formação/trajetória acadêmica hegemonicamente construída a partir das ciências biológicas passaram a investigar questões relacionadas ao aspecto comportamental da atividade física. Não há nada de errado com esta "migração" desde que quem há faça busque se apropriar da fundamentação necessária para o estudo do novo objeto. Importante mencionar que o contrário - pesquisadores com formação basicamente nas ciências humanas/sociais/comportamentais também podem "migrar" e passar a realizar suas pesquisas nas ciências biológicas, mas acredito que este caminho tem sido menos comum. De qualquer modo, este comentário sobre a "migração" dentro da "Atividade Física e Saúde" precisa ser ponderado, pois é apenas uma percepção individual. 


\section{Comentários sobre a produção em Atividade Física e Saúde no Brasil}

No momento atual parece existir uma supervalorização da importância do domínio das técnicas estatísticas. Não resta dúvida que o conhecimento destas técnicas pode ser de grande importância e ser um facilitador em vários sentidos. Muitos aprenderam (e bem) a "rodar dados", mas nem todos parecem ter profundidade para contribuir com um maior aprofundamento conceitual da área. Assim, muitos ao invés de compreenderem a estatística como uma ferramenta importante (e, portanto, meio) para a resolução de certas perguntas de pesquisa, passaram a entende-la como fim nela mesma. Sobre este tema, Hallal e Knuth (2011) ${ }^{13}$ destacam a importância de estudos qualitativos na Educação Física, e advogam a necessidade de uma maior aproximação entre os métodos quantitativos e qualitativos.

Além deste quase fetiche estatístico, é possível que haja entre alguns estudiosos a crença (errônea) que formular e aplicar questionários são tarefas simples. A noção de validação dos instrumentos também merece ser destacada. Alguns talvez acreditem que basta o questionário estar publicado para que este possa ser aplicado sem ressalvas, e desconsideram que outros aspectos precisam ser levados em conta na escolha dos instrumentos e técnicas de pesquisa. Apesar de não ser aprofundado este tema no presente texto - inclusive por fugir ao foco proposto - considero que um dos fatores que influenciou a "migração" de autores se deu pela noção que a aplicação de questionários é mais simples que outras técnicas para coleta de dados. Lembro que a maior parte dos estudos da "Epidemiologia da Atividade Física" publicados por autores brasileiros, fez uso de questionário como fonte principal de informação ${ }^{9}$.

Outro ponto relevante a ser abordado se dá pela discussão presente na Educação Física sobre a menor ou maior relação desta com as ciências humanas/sociais ou com as ciências da saúde/biológicas. Há de se reconhecer a influência de ambas. Rigo, Ribeiro e Hallal (2011) $)^{14}$ abordam esta temática e discutem a dificuldade de inserção internacional por parte dos trabalhos que apresentam maior aproximação com as ciências humanas/sociais, inclusive devido à dificuldade de replicação dos métodos e do interesse tender a ser mais localizado. Isto não quer dizer que estes estudos sejam menos relevantes que aqueles que conseguem inserção internacional. Os mesmos autores observam que enquanto o número de orientadores em programas de Pós-Graduação Stricto Sensu na área da Educação Física no Brasil, com ênfase na saúde ou ciências biológicas aumentou no período de 2007 a 2011, houve decréscimo no número destes que apresentavam maior relação com as ciências sociais/humanas. Apesar de compreender a divisão feita pelos autores (em ciências da saúde/biológicas $x$ ciências humanas/sociais), chamo a atenção para a grande diversidade na área "Atividade Física e Saúde", como mostrado na descrição das áreas de concentração e linhas de pesquisa dos programas de mestrado e doutorado (quadro 1).

\section{COMENTÁRIOS FINAIS}

Neste texto buscou-se discutir que atualmente, no Brasil, a área de "Atividade Física e Saúde" constitui-se hegemonicamente o que Kuhn chama de ciência normal (talvez uma "Nova ciência normal", como apontado na figura 1). Alguns autores que estudavam o desempenho humano, especialmente relacionado ao esporte hoje são também autores da "Atividade Física e Saúde". Lembro que os determinantes desta mudança podem estar mais relacionados à questões pragmáticas do que propriamente ao um convencimento generalizado que este objeto é mais relevante do que outros. Por questões pragmáticas entenda-se, neste momento, principalmente a perspectiva de publicação.

Neste sentido, considero a inserção de profissionais de Educação Física no Programa de Pós Graduação em Epidemiologia da UFPEL, a partir principalmente da década de $2000^{15}$, um momento chave, uma vez que a grande produção destes pesquisadores com formação inicial em Educação Física, chamou a atenção da área, e possivelmente "atraiu" novos pesquisadores para este paradigma. Evidentemente, este não foi o primeiro, nem o único momento relevante da história da "Atividade Física e Saúde" no Brasil ${ }^{16}$. Outros fatos contribuíram para chegarmos ao atual estágio, que parece ser de consolidação.

Vale mencionar que também outras áreas ou sub-áreas, como a Saúde Coletiva e a Epidemiologia, também questionam constantemente os seus próprios paradigmas. A leitura de Barata (2005) ${ }^{17}$, Susser e Susser ${ }^{18}$ e Almeida Filho, Castiel e Ayres ${ }^{19}$ ajuda a compreender a importância de questionamentos e inquietações, inclusive porque, para Kuhn o cientista normal não é preparado para ser crítico do paradigma em que trabalha, mas sim para resolver os "quebra-cabeças" que são pertinentes ao seu paradigma. Além do mais, se todos permanecessem cientistas normais, a ciência ficaria presa em um único paradigma e jamais progrediria para além deste.

Por outro lado, parece precipitado, e penso aqui estar o ponto central deste trabalho, considerar que "Atividade Física e Saúde" no Brasil seja uma "área" homogênea. Na verdade, existe uma parcela de autores que se identifica fundamentalmente com questões ligadas às ciências biológicas, enquanto que outros estão mais próximos das ciências sociais/humanas/comportamentais (muitos destes últimos influenciados principalmente pela Epidemiologia e pela Saúde Coletiva, como inclusive está explícito na descrição das linhas de Atividade Física e Saúde dos Programas da UFTM e da UPE/UFPB).

Independentemente da heterogeneidade, um ponto importante é que parecem faltar ainda "bandeiras comuns" à "Atividade Física e Saúde" no Brasil. Neste ponto, vale novamente a comparação com a Saúde Coletiva. Apesar de pontuais divergências, em alguns aspectos centrais parece haver consenso (por exemplo: na defesa do Sistema Único de Saúde, na importância dada aos valores democráticos na gestão em saúde, etc). Evidentemente que este aparente consenso da Saúde Coletiva, é fruto de uma construção coletiva, realizado principalmente a partir do chamado Movimento da Reforma Sanitária ${ }^{20}$. Na "Atividade Física e Saúde", pontos como a meIhoria da formação profissional para a atuação na saúde pública e a democratização da prática de atividade física poderiam de algum modo levar a uma maior unidade da área. É neste contexto, e com este ideário, que surge a Associação Brasileira para o Ensino da Educação Física para Saúde (ABENEFS) ${ }^{21}$, que se soma à outras instituições da área como as sociedades científicas já existentes, os grupos de pesquisa, entre outros.

Em relação ao futuro, esta é uma projeção difícil de ser realizada, mas concordo com Kuhn que a evolução do conhecimento científico se dá pela revolução e não pelo mero acúmulo. Particularmente, espero que a revolução se dê no sentido de se aproximar as explicações sobre os determinantes da prática de atividade física dos aspectos sociais, e que a área esteja comprometida com a melhoria da atuação profissional (que considero o grande problema da Educação Física brasileira) e com a melhoria (em um sentido amplo) da vida das pessoas. Neste ponto específico, concordo com Jenkins 


\begin{tabular}{|c|c|c|}
\hline Programa/IES & Característica & Descrição \\
\hline $\begin{array}{l}\text { Ciências da } \\
\text { Atividade } \\
\text { Física - } \\
\text { UNIVERSO }\end{array}$ & $\begin{array}{l}\text { Na área AF, Saúde e Sociedade } \\
\text { existem duas linhas de pesquisa: } \\
\text { Aspectos biodinâmicos da AF e } \\
\text { aspectos sócio culturais da AF. }\end{array}$ & $\begin{array}{l}\text { Aspectos biodinâmicos da } \mathrm{AF} \text { - Objetiva investigar os aspectos biológicos e biomecânicos das } \mathrm{AFs} \text {, } \\
\text { nas dimensões de prevenção, reabilitação e prescrição do exercício, na perspectiva da promoção da } \\
\text { saúde e do desempenho humano. } \\
\text { Aspectos Sócio culturais da } \mathrm{AF} \text { - Busca, a partir de uma perspectiva inclusiva/exclusiva investigar } \\
\text { os fenômenos sócio-culturais relacionados à atividade física, nas sua dimensões históricas, } \\
\text { sociológicas, administrativas, psicológicas e educacionais. }\end{array}$ \\
\hline $\mathrm{EF}-\mathrm{UCB}$ & $\begin{array}{l}\text { A área AF e Saúde divide-se em } \\
\text { três linhas: Aspectos Biológicos } \\
\text { Relacionados à AF e Saúde; } \\
\text { Aspectos Sócio-Culturais e } \\
\text { pedagógicos relacionados à AF e } \\
\text { Saúde; e Exercício Físico, } \\
\text { Reabilitação, Doenças Crônico não } \\
\text { Transmissíveis e Envelhecimento. }\end{array}$ & $\begin{array}{l}\text { Aspectos Biológicos Relacionados à AF e Saúde - Investiga as mudanças fisiológicas fundamentais } \\
\text { e as adaptações agudas e crônicas que ocorrem no corpo humano em diferentes tipos de exercício. } \\
\text { Aspectos Sócio-Culturais e pedagógicos relacionados à AF e Saúde - Caracteriza-se por estudos } \\
\text { relacionados à atividade física e saúde envolvendo aspectos sócio-culturais e pedagógicos. } \\
\text { Exercício Físico, Reabilitação, Doenças Crônico não Transmissíveis e Envelhecimento - } \\
\text { Caracteriza-se por estudos sobre a participação de exercício na prevenção e evolução de doenças } \\
\text { crônico não transmissíveis, na reabilitação e no envelhecimento. }\end{array}$ \\
\hline $\mathrm{EF}-\mathrm{USJT}$ & $\begin{array}{l}\text { Na área Escola, Esporte, AF e } \\
\text { Saúde existe a linha Promoção e } \\
\text { Prevenção em Saúde. }\end{array}$ & $\begin{array}{l}\text { Estuda a aplicação dos constructos básicos da Promoção da Saúde na perspectiva da Educação } \\
\text { Física, bem como desenvolve pesquisa relacionada à atividade física na Prevenção em Saúde em } \\
\text { vários níveis: fisiológico, morfológico, metabólico, perceptivo. Estuda, ainda, os resultados da } \\
\text { participação de diferentes populações em programas de educação física/atividade física quanto aos } \\
\text { aspectos antropológicos, biológicos, psicossociais, educacionais, sensoriais e motores. }\end{array}$ \\
\hline $\begin{array}{l}\text { Ciências da } \\
\text { Motricidade - } \\
\text { UNESP }\end{array}$ & $\begin{array}{l}\text { Na área Biodinâmica da } \\
\text { Motricidade Humana existe a } \\
\text { linha AF e saúde. }\end{array}$ & $\begin{array}{l}\text { Pesquisa os efeitos agudos, e em especial, os crônicos da AF, combinados ou não com estudo } \\
\text { farmacológico, nos componentes isolados ou multifatoriais determinantes da saúde, incluindo a } \\
\text { capacidade funcional e os diferentes estados patológicos, podendo associa-los com o processo de } \\
\text { envelhecimento. }\end{array}$ \\
\hline EF - UFRN & $\begin{array}{l}\text { Na área MH, Saúde e Desempenho, } \\
\text { existe a linha Avaliação e prescrição } \\
\text { na AF e saúde. }\end{array}$ & $\begin{array}{l}\text { Investigações de natureza biológica e comportamental com desdobramentos nas relações entre a } \\
\text { atividade física e seus efeitos agudos, sub-agudos e crônicos, em diferentes grupos populacionais e } \\
\text { contextos, como prevenção e promoção da saúde. }\end{array}$ \\
\hline \begin{tabular}{|l|} 
Ciências do \\
MH-UNICSUL \\
\end{tabular} & $\begin{array}{l}\text { Na área Biodinâmica do MH } \\
\text { existe uma linha AF e saúde. }\end{array}$ & $\begin{array}{l}\text { Estuda os efeitos do exercício físico e do nível de atividade física habitual na prevenção e/ ou } \\
\text { tratamento de desordens respiratórias, cardiovasculares e metabólicas. }\end{array}$ \\
\hline $\mathrm{EF}-\mathrm{UNB}$ & $\begin{array}{l}\text { Na área AF e Esporte existe a } \\
\text { linha Aspectos Biológicos } \\
\text { Relacionados ao Desempenho e à }\end{array}$ & $\begin{array}{l}\text { Investiga, de forma básica ou aplicada, as variáveis fisiológicas, biomecânicas e comportamentais } \\
\text { relacionadas ao desempenho e saúde decorrentes do desenvolvimento humano ou prática de } \\
\text { exercício e atividade física. }\end{array}$ \\
\hline EF - UNIMEP & $\begin{array}{l}\text { Na área MH, Cultura e Educação } \\
\text { existe a linha MH e Saúde. }\end{array}$ & $\begin{array}{l}\text { Estudos sobre o MH e suas potencialidades relativas à: a) promoção da qualidade de vida e da } \\
\text { saúde; b) desenvolvimento de métodos e técnicas para pesquisas e intervenção em EF e saúde e } \\
\text { estudos relativos ao metabolismo muscular, às relações enzimáticas e efeitos da suplementação } \\
\text { nutricional às respostas imunológicas na Performance Humana; c) Pesquisas relativas à natureza } \\
\text { humana e as respostas orgânicas gerais e neurofisiológicas e suas relações com a aptidão física e o } \\
\text { desenvolvimento humano, incluindo também populações de risco; d) observação de populações de } \\
\text { risco em prática de atividade física sistemática ou de lazer. }\end{array}$ \\
\hline \begin{tabular}{|l|} 
EF - \\
UEL/UEM
\end{tabular} & $\begin{array}{l}\mathrm{Na} \text { área Desempenho Humano e } \mathrm{AF} \\
\text { existe a linha AF relacionada à Saúde. }\end{array}$ & $\begin{array}{l}\text { Programas de atividade física em diversas populações, com emprego de métodos e técnicas de } \\
\text { avaliação de indicadores relacionados à saúde. }\end{array}$ \\
\hline EF - UFTM & $\begin{array}{l}\text { Na área Esporte e Exercício } \\
\text { existe a linha Esporte, Condições } \\
\text { de Vida e Saúde. }\end{array}$ & $\begin{array}{l}\text { Estuda a inter-relação da atividade física, aptidão física e saúde, por uma perspectiva } \\
\text { sociodemográfica e comportamental, apropriando-se dos referenciais da epidemiologia e da saúde } \\
\text { coletiva. Análise de aspectos do desenvolvimento motor em diferentes grupos populacionais } \\
\text { (portadores de necessidades especiais, gestantes, idosos). Programas educacionais e } \\
\text { desenvolvimento de recursos metodológicos para promoção de estilos de vida saudáveis. }\end{array}$ \\
\hline EF - UFES & $\mathrm{aEF}, \mathrm{MC}$ & $\begin{array}{l}\text { Estuda a relação do movimento corporal humano em suas diferentes manifestações socioculturais } \\
\text { com a saúde. }\end{array}$ \\
\hline $\mathrm{EF}-\mathrm{UFSC}$ & $\begin{array}{l}\text { AF relacionada à saúde é uma } \\
\text { área. Dentro dela, existem duas } \\
\text { linhas: Processos e Programas } \\
\text { de Promoção da AF e Saúde e } \\
\text { EF, Condições de Vida e Saúde. }\end{array}$ & $\begin{array}{l}\text { Processos e Programas de Promoção da AF - Fatores motivacionais para mudança de } \\
\text { comportamentos. Desenvolvimento de recursos para a promoção de um estilo de vida ativo. Meios } \\
\text { de avaliação nos programas de intervenção. Educação para um estilo de vida ativo na escola. } \\
\text { EF, Condições de Vida e Saúde - Relações da Educação Física com as condições de vida e de saúde } \\
\text { de diferentes grupos populacionais. Associação entre atividade física e indicadores gerais de saúde. } \\
\text { Educação em saúde nos diversos espaços de atuação da Educação Física. }\end{array}$ \\
\hline EF - UFPR & $\begin{array}{l}\mathrm{Na} \text { área Exercício e Esporte } \\
\text { existe a linha AF e Saúde. }\end{array}$ & $\begin{array}{l}\text { Análise dos aspectos da atividade física relacionados aos fatores que influenciam e determinam o } \\
\text { comportamento, estilo de vida e saúde. }\end{array}$ \\
\hline EF - UFPEL & $\begin{array}{l}\text { Na área AF, Saúde e } \\
\text { Desempenho existe a linha AF e } \\
\text { Saúde. }\end{array}$ & $\begin{array}{l}\text { O principal objetivo desta linha de pesquisa é estudar os efeitos da atividade física sobre a saúde } \\
\text { populacional. A linha de pesquisa também está interessada na avaliação e implementação de } \\
\text { intervenções em atividade física e discutir os fatores associados ao sedentarismo em nível } \\
\text { populacional, suas tendências temporais e associações com doenças crônicas relevantes no campo } \\
\text { da saúde pública. As estratégias de pesquisa dentro dessa linha são majoritariamente de cunho } \\
\text { epidemiológico, favorecendo o estudo de grupos populacionais. }\end{array}$ \\
\hline $\begin{array}{l}\text { EF- } \\
\text { UPE/UFPB }\end{array}$ & $\begin{array}{l}\text { Na área Saúde, Desempenho e } \\
\text { MV, existe a linha Inter-relação } \\
\text { AF e Saúde. }\end{array}$ & $\begin{array}{l}\text { Abrange investigações relativas à análise da inter-relação atividade física saúde por uma perspectiva } \\
\text { sociodemográfica, comportamental e biológica, apropriando-se do referencial teórico-metodológico } \\
\text { da epidemiologia e da saúde coletiva. Avaliação da efetividade e impacto de programas de } \\
\text { intervenção para promoção de estilo de vida saudável e prática de exercícios físicos em diferentes } \\
\text { grupos populacionais e contextos. }\end{array}$ \\
\hline $\begin{array}{l}\text { Ciências do MH } \\
\text { - UFRGS }\end{array}$ & $\begin{array}{l}\text { Na área MH, Saúde e } \\
\text { Performance existe uma linha } \\
\text { AF e Saúde. }\end{array}$ & $\begin{array}{l}\text { Compreende os projetos de pesquisa com desdobramentos nas relações entre o movimento humano } \\
\text { e seus efeitos preventivos e de reabilitação nas doenças relacionadas com as AFs. }\end{array}$ \\
\hline $\begin{array}{l}\text { Ciências do } \\
\text { MH- UDESC }\end{array}$ & $\begin{array}{l}\text { Na área Estudos } \\
\text { biocomportamentais do MH } \\
\text { existe uma linha AF e saúde. }\end{array}$ & $\begin{array}{l}\text { Estuda as implicações da AF na promoção, prevenção e manutenção da saúde e da qualidade de } \\
\text { vida. }\end{array}$ \\
\hline
\end{tabular}

Legendas: EF - Educação Física; AF - Atividade Física; MH: Movimento Humano; MCH: Movimento Corporal Humano.

UNIVERSO - Universidade Salgado de Oliveira; USJT - Universidade São Judas Tadeu; UNESP: Universidade Estadual Paulista Júlio de Mesquita Filho; UFRN: Universidade

Federal do Rio Grande do Norte; UNICSUL: Universidade Cruzeiro do Sul; UNB: Universidade de Brasília; UNIMEP: Universidade Metodista de Piracicaba; UEL/UEM:

Universidade Estadual de Londrina/Universidade Estadual de Maringá; UFTM: Universidade Federal do Triangulo Mineiro; UFES: Universidade Federal do Espirito Santo; UFSC:

Universidade Federal de Santa Catarina; UFPEL: Universidade Federal de Pelotas; UPE/UFPB: Universidade de Pernambuco/Universidade Federal da Paraiba; UFRGS: Universidade

Federal do Rio Grande do Sul; UDESC: Universidade do Estado de Santa Catarina. 
$(2007)^{22}$ quando este afirma que atualmente mais importante do que realização de novas descobertas nas ciências básicas, é a aplicação da psicologia e de outras ciências sociais e comportamentais para colocar em prática as medidas preventivas de saúde já comprovadas. Para tal, considero a inserção do profissional de Educação Física no Sistema Único de Saúde um fato de grande importância.

Entretanto, apesar deste desejo pessoal, tenho clareza, apoiado mais uma vez em Kuhn, que os rumos a serem tomados pela área dependerão fundamentalmente de aspectos pragmáticos. Assim, enquanto valores centrados no produtivismo acadêmico, que valoriza inclusive práticas condenáveis como a ciência salame, escambo autoral, entre outros ${ }^{23}$, forem supervalorizados em detrimento à outros aspectos (impacto social, impacto na atuação e formação profissional, etc), muito pouco será modificado. Sobre este tema, concordo com Waters (2006, p. 25) 24 :

"Estamos experimentando uma crise generalizada das avaliações, que resulta de expectativas não razoáveis sobre quantos textos um estudioso deve publicar. Não estou dizendo que não haja boas publicações - isso está muito longe de ser o caso -, mas o que as boas publicações têm de bom se perde em meio a tantas produções que são apenas competentes e muitas mais que não são nem isso. Protesto em nome dos bons livros que se perdem na enxurrada de livros ruins. E não estou dizendo que as coisas medianas não devem ser publicadas. Os estudiosos precisam escrever."

Este texto, muito mais do que concluir ou encerrar qualquer debate, buscou levantar elementos para a reflexão de alguns aspectos pertinentes à "Atividade Física e Saúde" no Brasil. Neste ponto, julgo ser necessário deixar explícito que considero a heterogeneidade constatada como potencialmente positiva. Existe uma interessante possibilidade de convivência entre diferentes olhares, que pode inclusive fazer a área crescer de maneira mais sólida. Por outro lado, reconhecer estas diferenças vai exigir maturidade da área, pois questões não resolvidas relativas às especificidades podem inclusive levar à questionamentos sobre a coerência de alguns projetos estarem "alocados" na Educação Física Sobre este tema, pondero que, considerando a história da Educação Física, bem como sua íntima relação com as "áreas mães", dificilmente um projeto será "puramente" da Educação Física, já que em alguma medida estará relacionado à alguma outra área (saúde coletiva, medicina, fisiologia, pedagogia, sociologia, etc.).

Outro ponto a ser destacado é a necessidade de reconhecimento da importância dos períodos de crise e revolução, pontos importantes da obra de Kuhn, especialmente para uma área nova e promissora, como é o caso da "Atividade Física e Saúde". Isso não significa desmerecer a importância dos períodos de "ciência normal". Compreender alguns elementos deste processo pode levar a um entendimento mais amplo (e crítico) de como se dá o processo de evolução do conhecimento científico e ajudar a área a se consolidar enquanto área acadêmica, bem como melhor lidar com suas diferenças internas.

\section{Agradecimento}

Ao professor Silvio Aparecido Fonseca pela leitura crítica da versão preliminar do texto.

\section{REFERÊNCIAS}

1. Kuhn T. A estrutura das Revoluções Científicas. São Paulo: Perspectiva; 2000.

2. Chalmers A. O que é ciência afinal? . São Paulo: Editora Brasiliense; 1993.

3. Popper K. Conjecturas e Refutações. Brasília: Ed. UNB; 1982.

4. Tani G. Os desafios da pós-graduação em educação física. Rev Bras Ciênc Es porte. 2000:;22(1):79-90

5. Kokubun E. Pós-graduação no Brasil: indicadores objetivos dos desafios e das perspectivas. Rev Bras Ciênc Esporte. 2003;24(2):9-26.

6. Betti M, Carvalho YM, Daolio J, Pires GL. A avaliação da educação física em debate: implicações para a subárea pedagógica e sociocultural. Revista Brasileira de Pós Graduação. 2004;1(2):183-94.

7. Rosa S, Leta J. Tendências atuais da pesquisa brasileira em Educação Física. Parte 2: a heterogeneidade epistemológica nos programas de pós graduação. Rev bras Educ Fis Esporte. 2011;25(1):7-18.

8. Bracht V. Educação Física e Ciência: cenas de um casamento infeliz. Ijuí: Editora Unijuí; 1999.

9. Hallal PC, Dumith SC, Bastos JP, Reichert FF, Siqueira FV, Azevedo MR. Evolução da pesquisa epidemiológica em atividade física no Brasil: revisão sistemática. Rev Saude Publica. 2007 Jun;41(3):453-60.

10. Dumith SC. Physical activity in Brazil: a systematic review. Cad Saude Publica. 2009;25 Suppl 3:S415-26.

11. http://conteudoweb.capes.gov.br/conteudoweb/ProjetoRelacaoCursosServl et?acao $=$ pesquisarles $\&$ codigo $A r e a=40900002 \&$ descricaoArea $=C \mathrm{Cl} C \mathrm{CANCIAS}$ $+\mathrm{DA}+\mathrm{SA} \% \mathrm{DADE}+$ \&descricaoAreaConhecimento=EDUCA\%C7\%C3O+F\%CDS CA\&descricaoAreaAvaliacao=EDUCA\%C7\%C3O+F\%CDSICA. Acessado em 20 de setembro de 2011

12. Carvalho DM. Epidemiologia - História e Fundamentos. In: Medronho R, editor Epidemiologia. São Paulo: Atheneu; 2006.

13. Hallal PC, Knuth AG. Epidemiologia da Atividade Física e a aproximação necessária com as pesquisas qualitativas. Rev Bras Ciênc Esporte 2011;33(1):181-92.

14. Rigo LC, Ribeiro GM, Hallal PC. Unidade na diversidade: desafios para a Educação Física no século XXI. Rev Bras de Atividade Física e Saúde 2011; 16(4): 339-345

15. Rodrigues CG, Loch MR, Garcia BG, Souza SC, editors. Inserção dos Profissionais de Educação Física em Programas de Pós-Graduação em Saúde Pública, Saúde Coletiva e Epidemiologia: Análise do período 2000-2009. Anais do XIX EAIC - Encontro Anual de Iniciação Científica da UNICENTRO; Guarapuava, PR 2010.

16. Nahas MV, Garcia LMT. Um pouco de história, desenvolvimentos recentes e perspectivas para a pesquisa em atividade física e saúde no Brasil. Rev bras Educ Fís Esp 2010;24(1):135-48.

17. Barata RB. Epidemiologia Social. Revista Brasileira de Epidemiologia. 2005;8(1):7-17.

18. Susser M, Susser E. Um futuro para a epidemiologia. In: Almeida Filho N, editor. Teoria epidemiológica hoje: fundamentos, interfaces, tendências. Rio de Janeiro: FIOCRUZ/ABRASCO; 1998.

19. Almeida Filho N, Castiel LD, Ayres JR. Riesgo: concepto básico de la epidemiología. Salud Colectiva. 2009:5(3):323-44

20. Paim J, Travassos C, Almeida C, Bahia L, Macinko J. The Brazilian health system: history, advances, and challenges. Lancet. 2011 May 21;377(9779):1778-97.

21. Fonseca SA, Menezes AS, Loch MR, Feitosa WMN, Nahas MV, Nascimento JV. Pela criação da Associação Brasileira de Ensino da Educação Física para a Saúde: ABENEFS. Revista Brasileira de Atividade Física e Saúde. 2011;16(4):283-8.

22. Jenkins $C D$. Construindo uma saúde melhor: um guia para mudança de comportamento. Porto Alegre: Artmed.; 2007.

23. Castiel LD, Sanz-Valero J. Entre fetichismo e sobrevivência: o artigo científi co é uma mercadoria acadêmica? Cad Saude Publica. 2007 Dec;23(12):3041-50.

24. Waters L. Inimigos da Esperança: publicar, perecer e o eclipse da erudição: Editora UNESP; 2006. 